\title{
EVOLUTIONARY COMPUTATION AND MODELING OF NATURAL PHENOMENA: AN APPLICATION TO LAVA FLOWS
}

\author{
William Spataro \\ Donato D'Ambrosio \\ Salvatore Di Gregorio \\ Department of Mathematics \\ University of Calabria \\ 87036 Rende (CS), Italy \\ E-mail: spataro@unical.it
}

\author{
Rocco Rongo \\ Maria Vittoria Avolio \\ Department of Earth Sciences \\ University of Calabria \\ 87036 Rende (CS), Italy \\ E-mail: rongo@unical.it
}

\author{
Giuseppe A. Trunfio \\ University of Sassari \\ Department of Architecture \\ and Planning \\ 07041 Alghero (SS), Italy \\ E-mail: trunfio@uniss.it
}

\section{KEYWORDS}

Cellular Automata, Genetic Algorithms, Modelling, Calibration, Validation, Parallel Computing, Simulation, Lava Flows

\begin{abstract}
A Parallel Master-Slave Genetic Algorithm has been applied to evolve a two dimensional Cellular Automata model for lava flow simulation. A new quantitative measure for the evaluation of the simulations result with respect to the real event has been defined and employed as fitness function. It compares both the areal extension and the temporal duration of the simulated event with respect the real one. The 2001 Etnean Nicolosi (Italy) case study has been considered for model calibration, while the validation has been performed by considering further cases of study, which differ both in duration and emission rate. Results have confirmed both the goodness of the simulation model and of the calibration algorithm. Eventually a possible application related to Civil Defence purposes is briefly described and proposed as a future development.
\end{abstract}

\section{INTRODUCTION}

Cellular Automata (CA) are discrete dynamical systems, widely utilized for modelling and simulating complex systems, whose evolution can be described in terms of local interactions. Well known examples are Lattice Gas Automata and Lattice Boltzmann models (Succi 2004), which are particularly suitable for modelling fluid dynamics at a microscopic scale. However, many natural phenomena are difficult to be modelled at such scale, as they generally evolve on very large areas, thus needing a macroscopic level of description. Moreover, they may be also difficult to be modelled through standard approaches, such as differential equations (McBirney and Murase 1984), and Macroscopic Cellular Automata (MCA) (Di Gregorio and Serra 1999) can represent a valid alternative.

Among the above mentioned phenomena, lava flows may involve serious dangers for people security and property, and their forecasting could significantly decrease this hazard, for instance by simulating lava paths and evaluating the effects of control works (e.g. embankments or channels). A crucial role is certainly covered by the simulation model, which must be characterized by an elevated degree of reliability. In other words, it must be properly calibrated, in order to reproduce a particular case of study at best, and validated on a sufficient number of different cases, in order to assess its goodness. SCIARA (Barca et al. 1994) is a family of deterministic MCA models, specifically developed for simulating lava flows, in particular for the Etnean "aa" type, which are characterized by a relatively high viscosity degree. Among the different releases, the last version, SCIARAfv, derived from SCIARA-hex1 (Crisci et al. 2004), was considered in this study, as in a first preliminary evaluation it demonstrated to be able to reproduce the qualitative behaviour of Etnean lava flows. Moreover, it demands for lower computational requirements with respect to its predecessors, which is particularly useful for the calibration phase, where an elevated number of simulations are generally needed.

In the next Sections, Macroscopic Cellular Automata and Genetic Algorithms are briefly presented. The first are considered in the simulation model definition, while the latter in its optimisation. Specifically, the MCA lava flows simulation model SCIARA-fv is illustrated, together with calibration results on the 2001 Etnean Nicolosi (Italy) case study. Subsequently, further cases of study are considered and results of model validation presented. Eventually, a possible application for Civil Defence purposes is discussed, and conclusions reported at the end.

\section{MACROSCOPIC CELLULAR AUTOMATA}

Macroscopic Cellular Automata were proposed by Di Gregorio and co-workers in 1982 to model the dynamics of macroscopic spatially extended systems, and firstly applied to the simulation of basaltic lava flows (Crisci et al. 1982). Subsequently, MCA were adopted for the simulation of many macroscopic phenomena, such as other kinds of lava flows (Crisci et al. 2004), debris flows (Iovine et al. 2005), as well as pyroclastic flows (Avolio et al. 2006), bioremediation processes (Di 
Gregorio et al. 2000) and traffic control (Di Gregorio et al. 1996).

With respect to the classical definition of Cellular Automata, MCA introduce some extensions. Firstly, the state of the cell is decomposed in "substates", each one representing a particular feature of the phenomenon to be modelled. The overall state of the cell is thus obtained as the Cartesian product of the considered substates. Moreover, some "parameters" are generally considered, which allow to "tune" the model for reproducing different dynamical behaviours of the phenomenon of interest. Eventually, as the cell state is subdivided in substates, even the state transition function is split in "elementary processes", each one describing a particular aspect of the considered phenomenon. Finally, several "external influences" can be considered in order to model features which are not easy to be described in terms of local interactions. An example of a MCA model for the simulation of lava flows is presented in the following sections.

\section{The Minimisation Algorithm of the Differences}

Phenomena involving flows which evolve at a macroscopic level of description are particular suitable to be modelled through MCA. If the cell dimension is a constant value throughout the cellular space, as usually occurs in MCA models, it is possible to consider characteristics of the cell (i.e. substates), typically expressed in terms of volume (e.g. lava volume), in terms of thickness. This simple assumption permits to adopt a straightforward but efficacious strategy that computes outflows from the central cell to the neighbouring ones in order to minimize the nonequilibrium conditions. Outflows computation is performed by the "minimisation algorithm of the differences", well described in (Di Gregorio and Serra 1999). It is based on the following assumptions:

- two parts of the considered quantity must be identified in the central cell: these are the unmovable part, $u(0)$, and the mobile part, $m$;

- only $m$ can be distributed to the adjacent cells. Let $f(x, y)$ denote the flow from cell $x$ to cell $y ; m$ can be written as:

$$
m=\sum_{i=0}^{\# X} f(0, i)
$$

where $f(0,0)$ is the part which is not distributed, and \#X is the number of cells belonging to the $X$ neighbourhood;

- the quantities in the adjacent cells, $u(i)(i=1,2, \ldots, \# X)$ are considered unmovable;

- let $c(i)=u(i)+f(0, i)(i=0,1, \ldots, \# X)$ be the new quantity content in the $i^{\text {th }}$ neighbouring cell after the distribution; let $c_{\min }$ be the minimum value of $c(i)(i=0,1, \ldots, \# X)$. The outflows are computed in order to minimise the following expression:

$$
\sum_{i=0}^{\# X}\left(c(i)-c_{\min }\right)
$$

The minimization algorithm operates as follows:

1. the following average is computed:

$$
a=\frac{m+\sum_{i \in A} u(i)}{\# A}
$$

where $A$ is the set of not eliminated cells (i.e. those that can receive a flow); note that at the first step $\# A=\# X$;

2. cells for which $u(i) \geq a(i=0,1, \ldots, \# X)$ are eliminated from the flow distribution and from the subsequent average computation;

3. the first two points are repeated until no cells are eliminated; eventually, the flow from the central cell towards the $i^{\text {th }}$ neighbour is computed as the difference between $u(i)$ and the last average value $a$ :

$$
f(0, i)= \begin{cases}a-u(i) & i \in A \\ 0 & i \notin A\end{cases}
$$

Note that the simultaneous application of the minimization principle to each cell gives rise to the global equilibrium of the system. The correctness of the algorithm is stated in (Di Gregorio and Serra 1999), i.e. it minimizes Equation (1).

\section{GENETIC ALGORITHMS}

Once a MCA model has been defined, it generally needs a calibration phase in order to individuate the parameters values which allow to reproduce the phenomenon at best. Even if no standardised optimisation techniques do exist for MCA, in previous works concerning debris and lava flows simulation (D'Ambrosio et al. 2006; Spataro et al. 2004), Genetic Algorithms (GAs) (Holland 1975) demonstrated to be a good choice.

Briefly, GAs are adaptive heuristic search algorithms inspired to Natural Selection and Genetics in which a solution to a given search problem is encoded as a genotype (or individual), and the set of all possible values it can assume is named search space. At the beginning, the GA randomly creates a population of individuals (candidate solutions), each one evaluated by means of a fitness function. Subsequently, the selection operator, which represents a metaphor of Darwinian Natural Selection, chooses individuals that undergo reproduction, by favouring the fittest ones (i.e. those having higher fitness). Reproduction is thus performed by means of genetic operators (generally crossover and mutation, representing a metaphor of sexual reproduction), and a new population of offspring obtained.

The evolution towards a good solution is typically obtained by the iterative application of selection and genetic operators to the initial population. The iterative process continues until one termination criterion is met, such as a known optimal or acceptable solution is attained, or the maximum number of steps is reached. 
The convergence to a good solution is stated by the "Fundamental Theorem of GAs" (Holland 1975).

\section{THE SCIARA-FV LAVA FLOWS SIMULATION MODEL}

As mentioned above, SCIARA-fv is the last release of a family of MCA models for lava flows simulation. The main model's characteristics can be summarised by the following points:

- it is a bi-dimensional model, based on hexagonal cells;

- the cell neighbourhood, $X$, is composed by the cell itself and the six adjacent ones;

- the model substates are $Q_{a}, Q_{t}, Q_{f}^{6}$ and $Q_{T}$ for altitude, lava thickness, lava flows from the central cell towards the six adjacent ones and temperature, respectively;

- lava feeding is modelled as an external influence by specifying cells which behave as vents;

- lava flows are computed by applying the minimisation algorithm of the differences, as described in the next section;

- lava temperature drop is modelled by applying the irradiation equation;

- lava viscosity varies according to lava temperature; it is modelled in terms of adherence, which specifies the amount of lava that cannot flow out of the cell;

- solidification process depends on lava temperature; it is trivially modelled by adding solidified lava thickness to the cell altitude.

Even though principally derived from the SCIARAhex1 version, SCIARA-fv embeds a better management of several aspects with respect to the original one, which will be described later.

In formal terms, SCIARA-fv is defined as

$$
\text { SCIARA-fv }=<R, L, X, Q, P, \tau, \gamma>
$$

where:

$-R$ is the set of hexagonal cells covering the finite region where the phenomenon evolves;

- $L \subset R$ specifies the lava source cells (i.e. vents);

- $X=\{$ Center, NW, NE, E, SE, SW, W $\}$ identifies the hexagonal pattern of cells that influence the cell state change. They are the cell itself, "Center", and the "North-West", "North-East", "East", "South-East", "South-West" and "West" neighbours;

- $Q=Q_{a} \times Q_{t} \times Q_{T} \times Q_{f}^{6}$ is the finite set of states, considered as Cartesian product of "substates". Their meanings are: cell altitude, cell lava thickness, cell lava temperature, and outflows lava thickness (from the central cell toward the six adjacent cells), respectively;

- $P=\left\{p_{s}, p_{T v}, p_{T s o l}, p_{a d v}, p_{a d s o l}, p_{\text {cool }}, p_{a}\right\}$ is the finite set of parameters (invariant in time and space), which affect the transition function; their meaning are: time corresponding to a CA step, lava temperature at the vent, lava temperature at solidification, lava adherence at the vent, lava adherence at solidification, the cooling parameter and cell apothem, respectively;

- $\tau: Q^{7} \rightarrow Q$ is the cell deterministic transition function;

- $\gamma: Q_{t} \times \mathbf{N} \rightarrow Q_{t}$ specifies the emitted lava thickness from the source cells at each step $k \in \mathbf{N}$ ( $\mathbf{N}$ is the set of Natural Numbers).

The transition function, in outline, is described in the following, in order of application.

\section{Lava flows computation}

Lava rheological resistance increases as temperature decreases; consequently, a certain amount of lava, i.e. the lava adherence $v$, cannot flow out from the central cell towards any neighbouring ones. It is obtained by means of the inverse exponential function:

$$
v=k_{1} e^{-k_{2} T}
$$

where $T \in Q_{T}$ is the lava temperature, while $k_{1}$ and $k_{2}$ are parameters depending on lava rheological properties (Park and Iversen 1984). The values for $k_{1}$ and $k_{2}$ are simply obtained by solving the equations system:

$$
\left\{\begin{array}{l}
p_{a d v}=k_{1} e^{-k_{2} p_{T V}} \\
p_{\text {adsol }}=k_{1} e^{-k_{2} p_{T s o l}}
\end{array}\right.
$$

Let $a \in Q_{a}$ and $t \in Q_{t}$ be the cell altitude and cell lava thickness, respectively; in order to compute lava outflows from the central cell towards its neighbouring ones, the minimisation algorithm is applied to the following quantities:

- $u(0)=a(0)+v$

- $m=t(0)-v$

- $u(i)=a(i)+t(i)(i=1,2, \ldots, 6)$

Eventually, a relaxation rate factor, related to the CA clock $p_{s}$ and to the cell size $p_{a}$, may be considered in order to obtain the local equilibrium condition in more than one CA step. This can significantly improve the realism of model as, in general, more than one step may be needed to displace the proper amount of lava from a cell towards the adjacent ones, especially when a small value for $p_{s}$ and high value for $p_{a}$ are considered. However, since a relatively high value for $p_{s}$ and a small value for $p_{a}$ were adopted in the simulations here presented, the relaxation rate was not taken into account in practice, and its exact specification thus omitted.

\section{Temperature Variation}

A two step process determines the new cell temperature. In the first one, the cell temperature is obtained as weighted average of residual lava inside the cell and lava inflows from neighbouring ones:

$$
T_{a v}=\left(t_{r} \times T(0)+\sum_{i=1}^{6} f(i, 0) \times T(i)\right) /\left(t_{r}+\sum_{i=1}^{6} f(i, 0)\right)
$$


where $t_{r} \in Q_{t}$ is the residual lava thickness inside the central cell after the outflows distribution, $T \in Q_{T}$ is the lava temperature and $f(i, 0)$ the lava inflow from the $i^{\text {th }}$ neighbouring cell. Note that $f(i, 0)$ is equal to the lava outflow from the $i^{\text {th }}$ neighbouring cell towards the central one, computed by means of the minimisation algorithm.

The final step updates the previous calculated temperature by considering thermal energy loss due to lava surface irradiation:

$$
T=T_{a v} / \sqrt[3]{1+\left(T_{a v}^{3} C A / V\right)}
$$

where $C=p_{\text {cool }}$ is the "cooling parameter" which depends on lava rheology, $A$ is the surface area of the cell, and $V$ the lava volume (Crisci et al. 2004).

\section{Lava Solidification}

When the lava temperature drops below the threshold $T_{\text {sol }}$, lava solidifies. Consequently, cell altitude increases by an amount equal to lava thickness and new lava thickness is set to zero.

\section{Software and Computational improvements}

With respect to previous versions, SCIARA-fv introduces many software improvements and computational optimizations, besides a thread-based multiple simulation feature.

As concerns software improvements, the source code was entirely rewritten in standard ANSI $\mathrm{C}++$ and an OpenGL 3D viewer added, which allows to monitor the evolution of a simulation in real time. These permit, among other matters, to have a fast and completely portable program, which can run both on Windows systems and Linux/Unix machines. Moreover, as lava flows may involve even a small sub-region of the overall considered area, a computational optimisation was introduced, which "restricts" the computation of the transition function to those cells belonging to the "minimum rectangular sub-area" containing all the "active cells" (i.e. those cells which contain lava - cf. Walter and Worsch 2004). The minimum rectangle is computed at each CA step, avoiding the computation over considerably wide regions of the cellular space, especially at the beginning of a simulation.

Table 1: Execution Times and Speedup of Benchmark Experiments Carried by adopting the SCIARA-fv Thread-based Multiple Simulation Feature on a NEC TX7 Supercomputer (having 16 Itanium Processors)

\begin{tabular}{ccc}
\hline Processors & Time & Speed-up \\
\hline 1 & 14.1 & 1 \\
2 & 7.1 & 1.97 \\
4 & 3.55 & 3.94 \\
8 & 1.775 & 7.88 \\
16 & 0.8875 & 15.77 \\
\hline
\end{tabular}

Finally, a threaded multiple simulation feature based on the portable OpenThreads cross-platform library, was implemented. It allows for the execution of multiple concurrent simulations, which can be useful for particular applications related to Civil Defence purposes, where an elevated number of experiments have to be carried out (cf. last section). Note that running many independent concurrent simulations permits to easily reach almost linear scalability (cf. Table 1), which may not be effortlessly achieved by parallelising the model itself. In other words, when the goal is the reduction of the execution time for a single experiment, the model parallelisation is preferable; on the other hand, when numerous simulations must be carried out in the less possible time, the concurrent execution of different sequential simulations is the best choice, as it generally guarantees better performances.

\section{SCIARA-FV CALIBRATION AND VALIDATION}

As previously stated, once that an MCA model has been defined, two stages are needed to assess its reliability: the calibration and validation phases. The former searches a set of parameters able to adequately reproduce a specific real case; the latter tests the model on a sufficient number of different cases (which should be different of those considered in the calibration phase, though similar in terms of physical and geological properties), permitting to give a final response on its goodness.

\section{SCIARA-fv Calibration}

As regards the calibration phase, it was performed by means of a genetic algorithm. It is similar to the that applied in Spataro et al. (2004), where parameters to be optimised were encoded as bit strings. Moreover, the GA is a steady-state and elitist model, so that at each step only the worst individuals are replaced. The remaining ones, required to form the new population, are copied from the old one, choosing the best. In order to select the individuals to be reproduced, the "binarytournament without replacement" selection operator was utilised. It consists of a series of "tournaments" in which two individuals are selected at random, and the winner is chosen according to a prefixed probability, which must be set greater for the fittest individual. In our case, this probability was set to 0.6. Moreover, as the variation without replacement scheme was adopted, individuals cannot be selected more than once. Employed genetic operators are classic Holland's crossover and mutation with probability of 1.0 and $2 / 44$, respectively. In particular, the above probability of mutation permitted to have, on an average, two bits mutated for each individual, as the genotype length (obtained as the sum of the number of bits chosen for the encoding of each considered SCIARA-fv parameters - cf. Table 2), was exactly 44. Eventually, the number of individuals forming the initial population was set to 256 , while the number of individuals to be replaced at each GA step was set to 16. Finally, the original fitness 
function $e_{1}$ (Spataro et al. 2004), was replaced with a new one.

The $e_{1}$ fitness function took into account only the comparison between the areal extensions of the real and simulated events; it was defined as:

$$
e_{1}=\sqrt{\frac{m(R \cap S)}{m(R \cup S)}}
$$

where $R$ and $S$ represent the areas affected by the real and simulated event, respectively, while $m(A)$ denotes the measure of the set $A$. Note that $e_{1} \in[0,1]$; its value is 0 if the real and simulated events are completely disjoint, being $m(R \cap S)=0$; it is 1 in case of perfect overlap, being $m(R \cap S)=m(R \cup S)$.

Preliminary calibration experiments were performed by adopting the original fitness function $e_{1}$. However, even if results seemed quite satisfactory for the considered case of study, it was not possible to well reproduce the further cases selected for the validation phase (especially in terms of run-out), mainly due to the fact that $e_{1}$ does not take into account any information about the duration of the real event. As a result, the best simulation gave its final shape at the end of the $8^{\text {th }}$ day, in spite of the $10^{\text {th }}$ needed for the real case. Thus, obtained parameters erroneously allowed for simulating a different kind of lava flows, e.g. characterised by a greater viscosity.

Table 2: The best set of SCIARA-fv parameters as obtained through calibration phase, together with their explored ranges. Note that parameter $p_{T v}$ was set to a

prefixed value, which corresponds to the typical temperature of Etnean lava flows at vents. Parameter $p_{a}$ was also prefixed, as it was imposed by the detail of the considered topographic data. The number of bits used

for the genetic algorithm encoding are also listed.

\begin{tabular}{cccc}
\hline Parameter & Explored range & Bits & Best value \\
\hline $\mathrm{p}_{\mathrm{s}}$ & {$[60,180]$} & 8 & $155.29 \mathrm{~s}$ \\
$\mathrm{p}_{\mathrm{Tv}}$ & - & - & $1373{ }^{\circ} \mathrm{K}$ \\
$\mathrm{p}_{\mathrm{Tsol}}$ & {$[1123,1173]$} & 8 & $1165.35^{\circ} \mathrm{K}$ \\
$\mathrm{p}_{\mathrm{adv}}$ & {$[0.1,2.0]$} & 4 & $0.7 \mathrm{~m}$ \\
$\mathrm{p}_{\mathrm{adsol}}$ & {$[6.0,30.0]$} & 6 & $12 \mathrm{~m}$ \\
$\mathrm{p}_{\text {cool }}$ & {$\left[10^{-16}, 10^{-13}\right]$} & 16 & $2.9 \cdot 10^{-14} \mathrm{~m}^{\circ} \mathrm{K}^{-3}$ \\
$\mathrm{p}_{\mathrm{a}}$ & - & - & $5 \mathrm{~m}$ \\
\hline
\end{tabular}

Hence, an improved fitness function, $f_{1}$, was devised, which takes into account both the areal extensions of the real and simulated events, and their temporal duration. It is defined as follows:

$$
f_{1}=\sqrt{e_{1}^{\left(t_{1}\right)} e_{1}^{\left(t_{2}\right)}}
$$

where $e_{1}$ is defined as before, while $t_{1}$ and $t_{2}$ represent two different temporal instants where it is evaluated. In particular, $t_{1}$ represents the time in which the real event reaches its stationary state. In this instant, the function $e_{1}$ is evaluated for the first time, giving information about the overlapping ratio of the simulation at that particular moment. However, contrarily to the real event, the simulation might not reach its final configuration at the same instant and its shape change further in time. In this case, if the function $e_{1}$ is again evaluated, for instance at the time $t_{2}>t_{1}$, its value could differ from the previous one, meaning that the overlapping ratio changed and thus the simulation did not stop when the real event did. Hence, as $e_{1}$ does, even the function $f_{1}$ gives values belonging to the interval $[0,1]$, with the difference that the value 1 is obtained when the real and simulated events perfectly overlap, with the further condition that the simulation stops exactly at the same time as the real event does. In other words, $f_{1}=1$ if and only if $e_{1}{ }^{(t 1)}=e_{1}{ }^{(t 2)}=1$.

Note that, by considering the available data concerning the cases of study here considered (limited to the areal extension and duration) $f_{1}$ can be considered a satisfying objective function for the model calibration phase. A more refined function can be certainly considered, e.g. by evaluating intermediate results along the overall period of evolution and not only at the end. However, its definition is constrained to the availability of reliable information about the real phenomenon, which is usually difficult to obtain.

Accordingly, the goal for the GA was to find a set of CA parameters that maximise $f_{1}$.

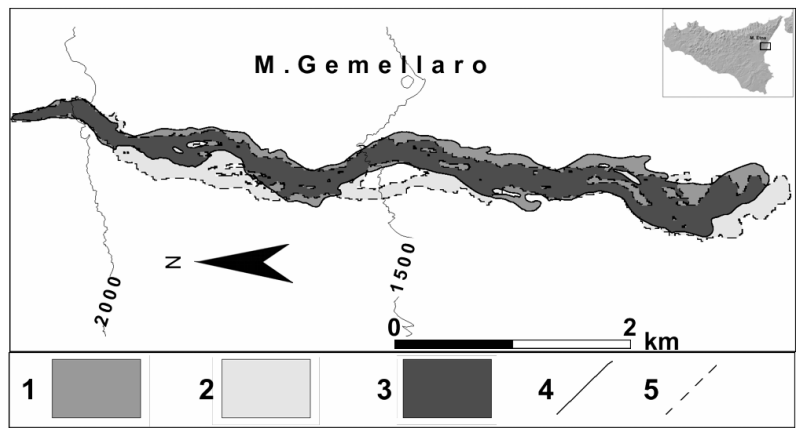

Figure 1a: Comparison between the 2001 Nicolosi Etnean Event and the Best SCIARA-fv Simulation, as obtained by Adopting the Parameters listed in Table 2.

Key: 1) Area affected by the Real event; 2) Area affected by the simulation; 3) Area affected by both Real and Simulated events; 4) Limits of Real event, 5) Limits of Simulated event.

The Case of Study and Calibration results. At 3.00 AM on July $18^{\text {th }}, 2001$, an eruption started from the fracture of Mount Calcarazzi, on the southern flank of Mt Etna (Sicily), $2100 \mathrm{~m}$ a.s.l. The event was fed by a medium lava flow rate (ca. $7 \mathrm{~m}^{3} / \mathrm{s}$ ) and, due to the steep descent of the terrain in that area, pointed southwards creating the main danger for the towns of Nicolosi and Belpasso. After 10 days of activity, it reached its maximum extension, which was almost 6 $\mathrm{Km}$ in terms of run-out. Such event was chosen as the reference case for the calibration phase, as it was considered sufficiently representative of Etnean lava 
flows and even characterised by a relative brief duration. In particular, the second feature allowed to execute the elevated number of simulations required by the GA in a reasonable amount of time. Moreover, to further speedup the experiments execution, a Master Slave parallel GA version was considered, instead of the sequential one, which simply split the individuals' fitness evaluation over the available "slave" processors, while the GA steps are managed by the "master" one. It represents the simplest example of Parallel Genetic Algorithm (Cantù-Paz 2000). Accordingly, calibration was performed on a Nec TX7 NUMA machine, composed by 4 quadri-processors Itanium class nodes, with an overall RAM memory of $32 \mathrm{~GB}$ and a peak performance of 64 GFLOPS.

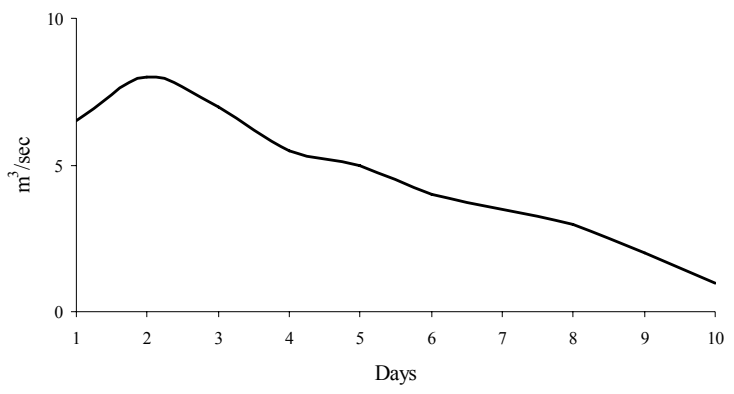

Figure 1b: Lava emission rate considered for the simulation illustrated in Figure 1a.

On the basis of previous empirical attempts, ranges within which the values of the $\mathrm{CA}$ parameters are allowed to vary were individuated in order to define the GA search space (cf. Table 2), and a set of 10 experiments iterated for 100 steps. As regards the fitness function, $t_{1}$ was set to 10 days (which corresponds to the duration of the real event), while $t_{2}$ was set to 13 days. The best result (cf. Figures 1a-b) allowed to satisfactorily reproduce the considered 2001 Nicolosi Etnean lava flow, giving rise to a fitness equal to 0.72 , which corresponds to a value of 0.74 in terms of areal comparison (i.e. in terms of $e_{1}$ ).

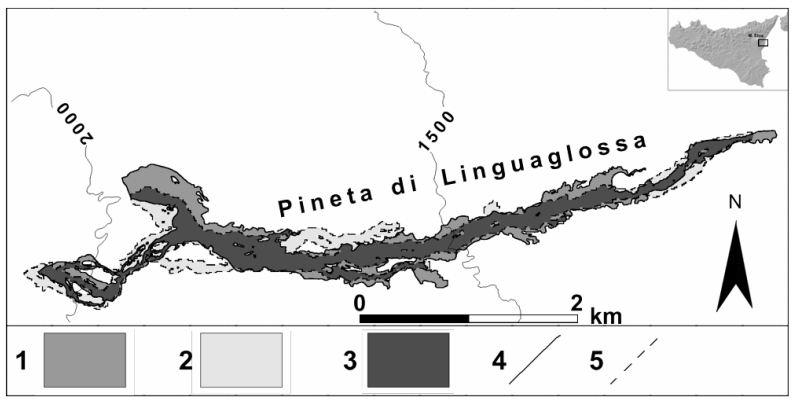

Figure 2a: Comparison between the 2002 Linguaglossa Etnean event and the SCIARA-fv simulation obtained by adopting the parameters listed in Table 2. Key: 1) area affected by the real event; 2) area affected by the simulation; 3 ) area affected by both real and simulated events; 4) limits of real event, 5) limits of simulated event.

\section{SCIARA-fv Validation}

A validation phase is generally needed in order to assess the goodness of parameters devised in the calibration phase, especially in case the fitness function only considers areal comparisons, as $e_{1}$. However, even if in this study a more refined function was preferred, which also considers a temporal comparison, calibration was performed on a "short" event (in terms of extension and duration), and results need to be confirmed on more general cases. As a consequence, the validation phase was carried out by testing the obtained parameters to other well-known real cases of study: the 2002 Linguaglossa and the 1991-93 Valle del Bove events, both regarding Mt Etna (Sicily). The first lasted 9 days, the second 473 days. Results are graphically illustrated in Figures 2a and 3a, respectively; Figures $2 \mathrm{~b}$ and $3 \mathrm{~b}$ show the corresponding emission rates. As expected, the best set of parameters (cf. Table 2) permitted a satisfactorily reproduction of the considered phenomena: in quantitative terms, the obtained $e_{1}$ values were 0.71 and 0.85 , respectively.

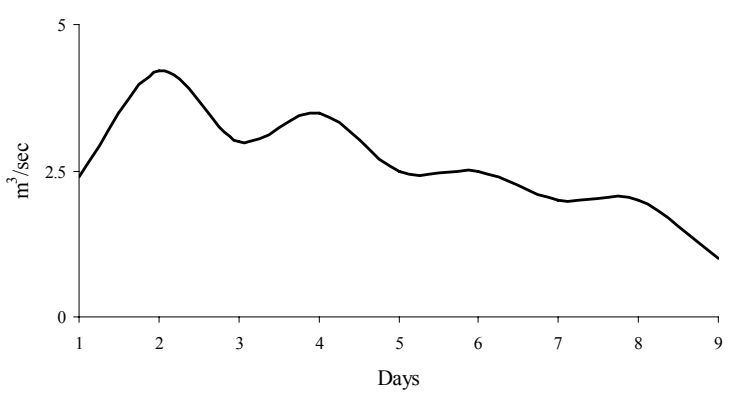

Figure 2b: Lava emission rate considered for the simulation illustrated in Figure 2a.

As a result, SCIARA-fv demonstrated to be able to simulate Etnean lava flows, also showing a satisfactorily ability to reproduce lava fields (cf. Figure 3a). However, two further experiments were performed for better assessing such ability. Accordingly, the Nicolosi lava flow was left to evolve for further 90 days (for a total of 100 days), and a final simulation performed by considering a vent near the one activated in the 1792 Etnean lava flow and left to evolve for 90 days (which correspond to the duration of the 1792 eruption). Note that, in the latter case, pre-event morphological data was obviously not available and a comparison with the real event not possible.

Good results were obtained in both cases (cf. Figures 4 and $5 \mathrm{a}-\mathrm{b}$ ), permitting to give a final response on the model's ability in reproducing lava fields and, more in general, in reproducing Etnean lava flows. Thus, on the basis of the above results and by considering that Etnean lava flows may be essentially considered as characterised by the same rheological features (Chester et al. 1985), the MCA model SCIARA-fv could be confidently adopted for simulating new cases on the same study area. 


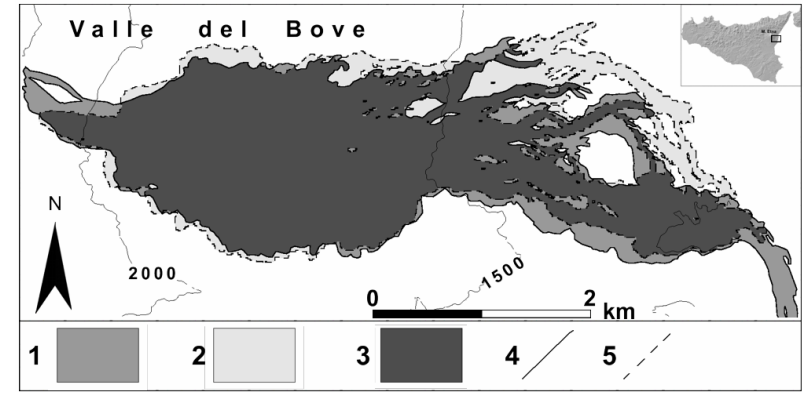

Figure 3a: Comparison between the 1991-93 Valle del Bove Etnean event and the SCIARA-fv simulation obtained by adopting the parameters listed in Table 2 . Key: 1) area affected by the real event; 2) area affected by the simulation; 3 ) area affected by both real and simulated events; 4) limits of real event, 5) limits of simulated event.

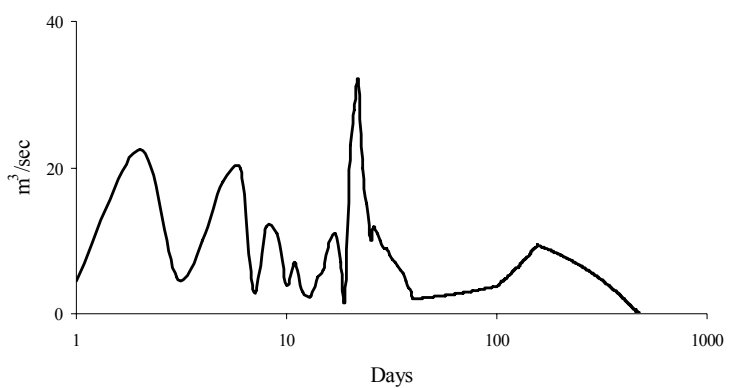

Figure 3b: Lava emission rate considered for the simulation illustrated in Figure 3a. Note that the Days axis is in logarithmic scale.

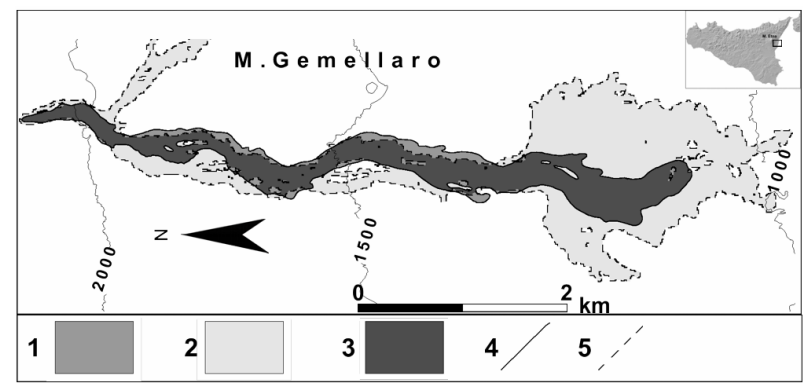

Figure 4: Comparison between the 2001 Nicolosi Etnean event and the SCIARA-fv simulation obtained by adopting the parameters listed in Table 2 . The simulation was iterated for 100 days (instead of 10, i.e. the duration of the real event), by considering a constant emission rate of $12 \mathrm{~m}^{3} / \mathrm{s}$. A wide lava field between the altitudes 1250 and $100 \mathrm{~m}$ a.s.l. is well visible. Key: 1) area affected by the real event; 2) area affected by the simulation; 3 ) area affected by both real and simulated events; 4) limits of real event, 5) limits of simulated event.

\section{A POSSIBLE APPLICATION FOR CIVIL DEFENCE PURPOSES}

Once SCIARA-fv has been well calibrated and validated, and its reliability in well simulating Etnean lava flows assessed, a possible application related to Civil Defence purposes can be conjectured. In this work, a methodology for the definition of a new kind of map showing the hazard related to lava invasion in predefined study areas is proposed.

Differently to standard approaches, in which the hazard is generally based on statistical studies of past events (Behncke et al. 2005), the one here proposed relies on a "virtual laboratory" (i.e. the SCIARA-fv framework) where new events are simulated on present morphological data, which implicitly embeds the effects of past events.

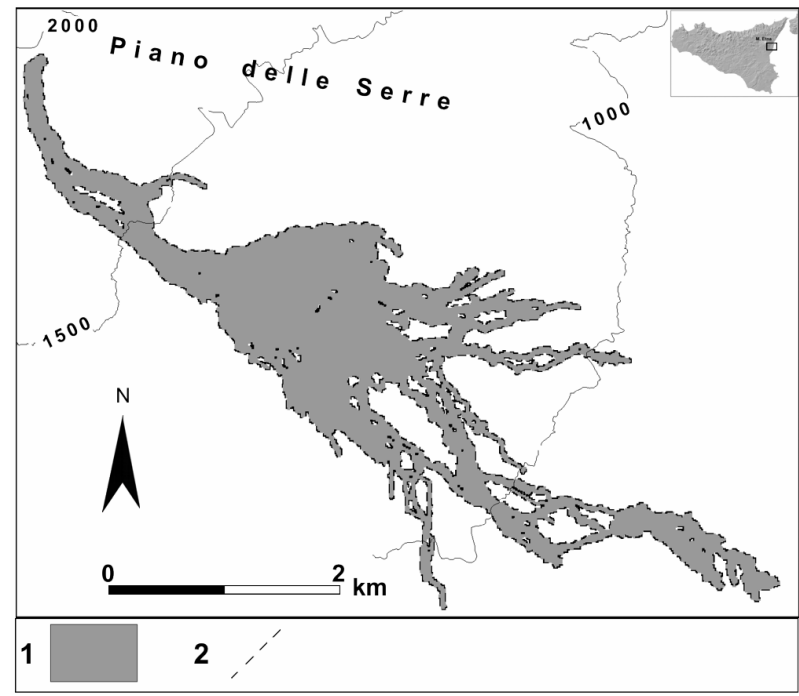

Figure 5a: Simulation performed by activating a vent near the one opened in the 1792 Etnean lava flow and left to evolve for 90 days. A wide lava field between the altitudes 1400 and $1000 \mathrm{~m}$ a.s.l. is well visible. Key: 1) area affected by the simulation; 2) limits of simulated event.

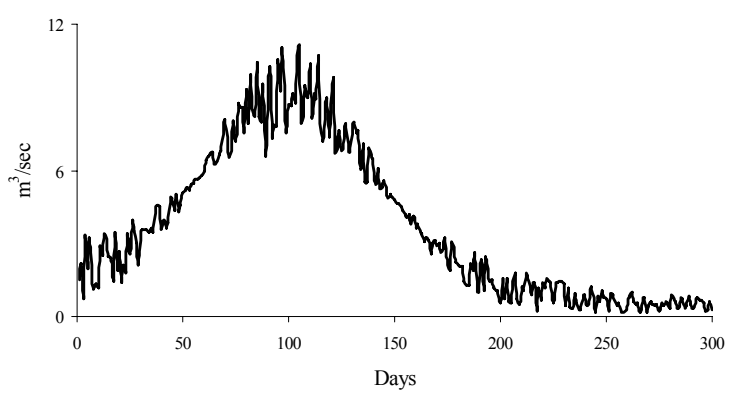

Figure 5b: Lava emission rate considered for the simulation illustrated in Figure 5a.

A grid of vents can be defined in the study area, and a prefixed number of simulations executed for each of them, each one characterised by its own emission rate and duration. Moreover, a probability of activation can be assigned to each vent in the grid (activation probability), based on historical, prehistoric and geological data, and a probability assigned to each type of considered emission rate and duration (event 
probability), devised on the basis of the emission behaviour analysis of the study area.

Eventually, on the basis of other considerations, more additional probabilities can be considered (e.g. a higher probability can be assigned to an event on the basis of the minor distance of the vent with respect to the summit craters - altitude probability). The resulting hazard map is thus compiled by taking into account both information on lava flows overlapping, and their occurrence probability.

Accordingly, a simulation is executed for each combination of vent location and event history, by storing results in a database. The resulting map is obtained by evaluating the hazard at each point in the study area as follows: 1) for each simulation, the hazard related to a point is computed as the product of the defined probabilities of occurrence (conditioned probability) if it is affected by the simulated lava flow, zero otherwise; 2) for each point, the conditioned probabilities are added over all the performed simulations. Note that, in such a way some areas will be characterised by very low hazard values (even zero), while others by high ones. Depending on the number of performed simulations and morphological conditions, the hazard of remaining areas may range in a quasicontinuous manner between the two extremes. As a consequence, it may be possible to compile hazard maps with a high level of description even if, in general, few hazard classes are considered adequate for many practical applications.

The accuracy of the results strictly depends on the reliability of the simulation model, on the quality of input data and on the hypotheses on assigning the different probabilities of occurrence. Thus, if some of such aspects should not be sufficiently adequate, it could be possible (and desirable) to improve them in order to compile a resulting hazard map with a higher level of accuracy. For instance, in case of uncertainty in assigning the probabilities of occurrence, a different map can be obtained by simply re-processing the simulations database and by just considering a more reliable criterion of analysis. Finally, note that if an equal probability of occurrence is assigned to each simulation, a more classical criterion of hazard mapping is obtained, which only considers the number of simulated events which affect a given area.

At present, this methodology is a work in progress and it is being applied to the definition of a hazard map for the South-Eastern flank of Mt Etna. Figure 6 shows the considered area, together with the location of vents subdivided in 4 classes of activation probabilities, these latter derived by a statistical study on lava events occurred over the last 400 years and on geological considerations (Behncke et al. 2005). The grid of vents consists of 340 points, each one located $250 \mathrm{~m}$ apart from each other, in order to uniformly cover the interested area.

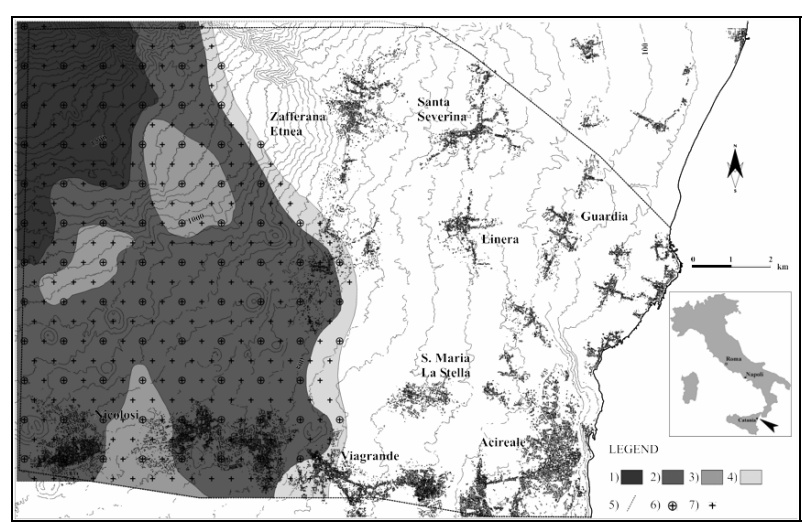

Figure 6: The South-Eastern flank of Mt Etna. Key: 1-4) vent activation probability, in decreasing order, corresponding to $4 / 10,3 / 10,2 / 10$ and $1 / 10$, respectively; 5) limits of the study area; 6 ) the 88 vents considered in the present work; 7) remaining 252 vents.

As regards the simulation phase, 50 events have been chosen for each of the 340 considered vents (cf. keys 6 and 7, Figure 6), and therefore a total of 17000 experiments have to be executed for an exhaustive study.

By considering the extent of the study area (a map of $2272 \times 1790$ hexagonal cells, each with a $5 \mathrm{~m}$ apothem, derived from a 1:10000 scale topography) and the duration of the considered events (which ranges from 15 to 500 days), the adoption of Parallel Computing is mandatory to reduce the execution time. In this phase, the thread-based simulation feature of SCIARA-fv is crucial (it allows for almost linear speedup) to perform all the required experiments in a reasonable amount of time. Simulations are still underway. However, the number of carried out experiments is still too low to permit a significant hazard mapping for the considered study area. Therefore, final results are here omitted and postponed to a future work.

\section{CONCLUSIONS}

We proposed the application of a Master Slave GA to the calibration of a new MCA model for lava flows simulation, namely SCIARA-fv, by considering the Etnean 2001 Nicolosi (Italy) case study. A new fitness function, $f_{1}$, which compares both the areal extension and the temporal duration of the simulated event with respect the real one, has been introduced and adopted in spite the original one, $e_{1}$, which took into account only an areal comparison.

A validation phase was also carried out on different cases of study, demonstrating both the GA's reliability, and the SCIARA-fv efficacy in the simulation of Etnean lava flows.

From a qualitative point of view, all the simulations carried out by considering MCA parameters corresponding to the best evolved individual do not differ significantly from the real cases, which is also confirmed by good values in terms of the function $e_{1}$. 
Moreover, a new kind of criterion for the compilation of lava invasion susceptibly maps has been proposed. Results referred to the application to the South-Eastern flank of Mt Etna, even if preliminary, seemed to confirm the goodness of the approach. Nevertheless, results have been omitted as the number of performed simulations allowed only for a qualitative evaluation. This study is currently a work in progress, and final results will be presented in a future publication.

However, a more rigorous assessment of the reliability of the proposed methodology is certainly desirable for effective usage in Civil Defence. A possible solution could simply consist awaiting for next events in the study area but this could, obviously, require an unpredictable time. An alternative could consist in compiling the map on a subset of sample events (e.g. occurred in the first 300 years) and validate it over the remaining ones, with the condition to dispose of a proper past topography.

Other alternatives are also currently being conjectured, which will be certainly taken into account in future works.

\section{ACKNOWLEDGEMENTS}

This work is sponsored by the Italian Ministry for Education, University and Research, FIRB project $\mathrm{n}^{\circ}$ RBAU01RMZ4 "Lava flow simulations by Cellular Automata". Geological data, lava flows emission rates, and topographic maps have been provided by Dr. S. Calvari, Dr. M. Neri and Dr. B. Behncke of the INGV (National Institute for Geophysics and Volcanology) of Catania (Italy). Note that the $f v$ suffix in the SCIARA name stands for "FIRB Version". The authors are also grateful to Prof. Gino Mirocle Crisci, Dr. Giulio Iovine, Dr. Valeria Lupiano and Dr. Giancarlo Niceforo for the common researches.

\section{REFERENCES}

Avolio, M.V.; G.M. Crisci; S. Di Gregorio; R. Rongo; W. Spataro; and D. D'Ambrosio. 2006. "Pyroclastic flows modelling using cellular automata." Computers and Geosciences-UK, 32, 897-911.

Barca, D.; G.M. Crisci; S. Di Gregorio; and F. Nicoletta. 1994. "Cellular Automata for simulating lava Flows: A method and examples of the Etnean eruptions." Transport Theory and Statistical Physics, Vol.23, 195-232.

Behncke, B.; M. Neri; and A. Nagay. 2005. "Lava flow hazard at Mount Etna (Italy): New data from a GIS-based study." In Kinematics and dynamics of lava flows: Geological Society of America Special Paper, M. Manga, and G. Ventura (Eds.), Vol.396, 189-208.

Cantù-Paz, E. 2000. Efficient and accurate Parallel Genetic Algorithms, Kluwer Academic Publishers, Dordrecht, The Netherlands.

Chester, D.K.; A.M. Duncan; J.E. Guest; and C.R.J. Kilburn. 1985. Mount Etna: The anatomy of a volcano, Chapman and Hall, London.

Crisci, G.M.; R. Rongo; S. Di Gregorio; and W. Spataro. 2004. "The simulation model SCIARA: the 1991 and 2001 lava flows at Mount Etna." Journal of Volcanology and Geothermal Research, Vol.132, 253-267.

Crisci, G.M.; S. Di Gregorio; and G.A. Ranieri. 1982. "A cellular space model of basaltic lava flow." In Proceedings International AMSE Conference Modelling \& Simulation, (Paris, France, Jul.1-3). 65-67.

D'Ambrosio, D.; W. Spataro; and G. Iovine. 2006. "Parallel genetic algorithms for optimising cellular automata models of natural complex phenomena: an application to debris-flows". Computers and Geosciences-UK, 32, 861-875.

Di Gregorio S.; D.C. Festa; R. Rongo; W. Spataro; G. Spezzano; and D. Talia. 1983. "A microscopic freeway traffic simulator on a highly parallel system." In Parallel Computing: State-ofthe-Art and Perspectives, E.H. D'Hollander, G.R. Joubert, F.J. Peters and D. Trystam (Eds.), 69-76.

Di Gregorio, S. and R. Serra. 1999. "An empirical method for modelling and simulating some complex macroscopic phenomena by cellular automata." Future Generation Computer Systems, Vol.16, 259-271.

Di Gregorio, S.; R. Serra; and M. Villani. 1997. "A Cellular Automata Model of Soil Bioremediation." Complex Systems Vol.11, 31-54.

Holland, J.H. 1975. Adaption in Natural and Artificial Systems, University of Michigan Press, Ann Harbor.

Iovine, G.; D. D’Ambrosio; and S. Di Gregorio. 1981. “Applying genetic algorithms for calibrating a hexagonal cellular automata model for the simulation of debris flows characterised by strong inertial effects." Geomorphology, Vol.66, 287-303.

McBirney, A.R. and T. Murase. 1984. "Rheological properties of magmas." Annual Review of Earth Planetary Sciences, Vol.12, 337-357.

Park, S. and J.D. Iversen. 1984. "Dynamics of lava flow: Thickness growth characteristics of steady two-dimensional flows." Geophysical Research Letters, Vol.11, 641-644.

Spataro, W.; D. D’Ambrosio; R. Rongo; and G.A. Trunfio. 2004. "An Evolutionary Approach for Modelling Lava Flows through Cellular Automata." In Proceedings of the 7th International Conference on Cellular Automata for Research and Industry (Perpignan, France, Sep.20-23). LNCS 4173, $725-734$.

Succi, S. 2004. The Lattice Boltzmann Equation for Fluid Dynamics and Beyond. Oxford University Press.

Walter, R. and T. Worsch. 2004. "Efficient Simulation of CA with Few Activities." In Proceedings of the $6^{\text {th }}$ International Conference on Cellular Automata for Research and Industry (Amsterdam, The Netherlands, Oct.25-27), LNCS 3305, 101110.

\section{AUTHOR BIOGRAPHIES}

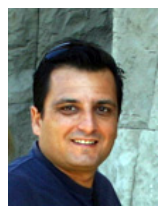

WILLIAM SPATARO was born in London, UK. He is an Assistant Professor in Computer Science at the Department of Mathematics at the University of Calabria (Italy). His principal interests regard Parallel Computing, Cellular Automata, Modelling and Simulation of complex phenomena and Genetic Algorithms. His e-mail address is: spataro@unical.it and his Web-page can be found at http://www.mat. unical.it/spataro.

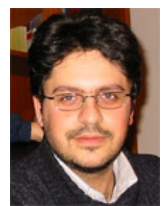

DONATO D'AMBROSIO was born in Cosenza, Italy. He is an Assistant Professor in Computer Science at the Department of Mathematics at the University of Calabria 
(Italy). His interests concern Genetic Algorithms, Parallel Computing, Simulation of Complex Natural Phenomena and Computer Graphics. His e-mail address is: d.dambrosiodunical.it and his Web-page can be found at http://www.mat.unical.it/ donato.

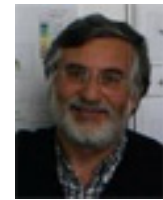

SALVATORE DI GREGORIO was born in Castellamare del Golfo, Sicily, Italy. He is Full Professor in Computer Science at the Department of Mathematics at the University of Calabria (Italy). Together with Professor Gino Mirocle Crisci, he leads the Empedocles Research group at the same University. His research interests concern Computational Models of Complex Systems and Parallel Computing. His e-mail address is: dig@unical.it.

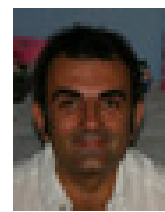

ROCCO RONGO was born in Naples, Italy. $\mathrm{He}$ is an Assistant Professor in Computer Science at the Department of Earth Sciences at the University of Calabria (Italy). His major interests regard Parallel Computing, Modelling and Simulation of Geological processes by Cellular Automata and GIS software. His e-mail address is: rongoeunical.it and his Web-page can be found at http://dister.unical.it/rongo/.

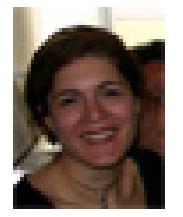

MARIA VITTORIA AVOLIO was born in Cosenza, Italy. Mathematician and Researcher Assistant at the Department of Earth Sciences at the University of Calabria, Italy, she is involved basically in Modelling and Simulation of lava, landslide and pyroclastic flows by Cellular Automata methods. She is the beloved wife of William Spataro, who fell in love with her during her $\mathrm{PhD}$ course in Computer Science and Mathematics at the same University. Her e-mail address is: avoliomv@unical.it.

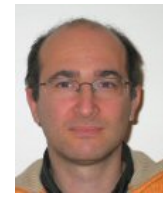

GIUSEPPE A. TRUNFIO gained a Ph.D. in Computational Mechanics in 1999 at the University of Calabria, Italy. He has been a research fellow at the Italian National Research Council where he has worked extensively on the application of parallel computing to the simulation of complex systems. He is Assistant Professor of Computer Engineering at the Department of Architecture and Planning of the University of Sassari and his current research interests include decision support, probabilistic models, neural networks, evolutionary computation and cellular automata. His email address is: trunfio@uniss.it. 